\title{
Sex Steroid Metabolism in Benign and Malignant Intact Prostate Biopsies: Individual Profiling of Prostate Intracrinology
}

\author{
Daniele Gianfrilli, ${ }^{1}$ Silvia Pierotti, ${ }^{1}$ Riccardo Pofi, ${ }^{1}$ Costantino Leonardo, \\ Mauro Ciccariello, ${ }^{3}$ and Federica Barbagallo ${ }^{1}$ \\ ${ }^{1}$ Department of Experimental Medicine, Sapienza University, Viale del Policlinico 155A, 00161 Rome, Italy \\ ${ }^{2}$ Department of Urology, Sapienza University, 00161 Rome, Italy \\ ${ }^{3}$ Department of Radiology, Sapienza University, 00161 Rome, Italy
}

Correspondence should be addressed to Daniele Gianfrilli; daniele.gianfrilli@uniromal.it

Received 2 May 2014; Accepted 18 June 2014; Published 13 August 2014

Academic Editor: Giovanni Luca Gravina

Copyright (C) 2014 Daniele Gianfrilli et al. This is an open access article distributed under the Creative Commons Attribution License, which permits unrestricted use, distribution, and reproduction in any medium, provided the original work is properly cited.

\begin{abstract}
In vitro studies reveal that androgens, oestrogens, and their metabolites play a crucial role in prostate homeostasis. Most of the studies evaluated intraprostatic hormone metabolism using cell lines or preprocessed specimens. Using an ex vivo model of intact tissue cultures with preserved architecture, we characterized the enzymatic profile of biopsies from patients with benign prostatic hyperplasia (BPH) or cancer (PC), focusing on $17 \beta$-hydroxy-steroid-dehydrogenases (17 $\beta$-HSDs) and aromatase activities. Samples from 26 men who underwent prostate needle core biopsies (BPH $n=14 ; \mathrm{PC} n=12$ ) were incubated with radiolabeled ${ }^{3} \mathrm{H}$-testosterone or ${ }^{3} \mathrm{H}$-androstenedione. Conversion was evaluated by TLC separation and beta-scanning of extracted supernatants. We identified three major patterns of conversion. The majority of BPHs revealed no active testosterone/oestradiol conversion as opposed to prostate cancer. Conversion correlated with histology and PSA, but not circulating hormones. Highest Gleason scores had a higher androstenedion-to-testosterone conversion and expression of 17 $\beta$-HSD-isoenzymes-3/5. Conclusions. We developed an easy tool to profile individual intraprostatic enzymatic activity by characterizing conversion pathways in an intact tissue environment. In fresh biopsies we found that $17 \beta$-HSD-isoenzymes and aromatase activities correlate with biological behaviour allowing for morphofunctional phenotyping of pathology specimens and clinical monitoring of novel enzyme-targeting drugs.
\end{abstract}

\section{Introduction}

Prostate cancer is the most common cancer in men. An increasing trend in prostate cancer incidence, a disease associated with age, has been described and partially attributed to better screening procedures [1] and awareness [2]. A significant number of prostate cancers, however, remain indolent and, if untreated, do not alter life quality and expectancy. For this reason the burden of universal treatment of confined asymptomatic disease should be weighed against the economic socioeconomic costs of overtreatment, the complications associated with the currently available treatments (including androgen-deprivation therapy), and the overall quality/life expectancy of affected subjects $[3,4]$.

Improvements in prostate cancer diagnosis, classification, and treatment witnessed in the past 20 years have not been paralleled by improvement in preoperative prognostic grading of the disease which still relies on morphological appearance of random biopsies. A functional prognostic presurgical characterization of the disease is needed to identify those subjects who require aggressive treatment and those who can be managed conservatively. Furthermore, a function profiling of prostate tissue will also be very useful to monitor unoperated patients during radiotherapy (RT) and androgen-deprivation therapy (ADT) to follow up changes in prostate tissue responsiveness and aggressiveness.

It is widely accepted that androgens play a central role in the biology of the prostate. Estrogens, however, can also modulate prostatic growth and development $[5,6]$. Taken together, observations from many studies on murine models imply that both androgens and estrogens are needed to induce proliferative, precancerous lesions and prostate 
cancer. Indeed, the balance between androgen induced cell proliferation and apoptosis is thought to be a major regulator of growth of the normal and cancerous prostate. Epidemiological studies, however, showed that there is no association between circulating steroid hormone levels and prostate cancer [7]. In vitro studies reveal that intratissual levels of sex steroids may diverge from their plasmatic counterpart due to complex enzymatic equipment expressed by prostate cells that can interconvert steroids [8]. Labrie et al. was the first to describe the "intracrinology" of the prostate gland $[9,10]$.

Prostate tissue contains a variety of steroid metabolizing enzymes required for the local production of active androgens and estrogens from their precursors provided by the adrenals [10-12]. The main enzymes involved in local steroid metabolism are steroid sulfatases, $3 \beta$-hydroxysteroid dehydrogenases ( $3 \beta$-HSDs), $17 \beta$-hydroxysteroid dehydrogenases (17 $\beta$-HSDs), $5 \beta$-reductases, and aromatase. In normal conditions a steady state exists between synthesis and inactivation of active androgens; however tissue transformation can be associated with an alteration of this balance. Increasing evidences suggest that prostate cancer cells alter local and paracrine steroid hormone metabolism. In the past decade, a growing number of studies tried to explore the role of local androgen production in cancer progression and transformation into castrate-resistant tumours (CRPC) $[13,14]$.

In the present work we provide evidences that intraprostatic hormonal profiling, some sort of individual metabolic fingerprint, can be easily obtained. One of the most innovative features of the present study is that we analysed the metabolism of prostate cells directly "ex vivo" on fresh specimens from biopsy or surgical resection. The aim of the current work is (1) to set up a reproducible, rapid, and easy approach to define the enzymatic profiling of the normal, hyperplastic (BPH), and cancerous prostate cell (PC) (2) to correlate the patterns with steroid enzymes' expression and tumour's histology.

\section{Methods}

2.1. Patients and Tissues. Specimens were obtained from 26 patients (14 $\mathrm{BPH}$ and $12 \mathrm{PC}$ ) who underwent transrectal ultrasound-guided prostate biopsy followed by prostate surgery (radical for cancer or transurethral for enlargement) between 2009 and 2012 at the Department of Urology, Sapienza University. The clinical characteristics are reported in Table 1.

All patients examined in this study did not receive radiation, chemotherapy, or hormone therapy before surgery. Clinical data, including patient age, serum prostate specific antigen (PSA) concentration, clinical stage according to the International TNM classification, lymph node status, and Gleason's score, were retrieved for all patients. All procedures were performed using commercially available ultrasound equipment with 7.5 MHz probes (Philips IU22); biopsy samples were obtained using an automatic spring-loaded biopsy gun with an 18-gauge needle.

Specimens upon collection were placed on saline buffer and immediately processed (Figure 1). The protocol was
TABLE 1: Characteristics of enrolled subjects.

\begin{tabular}{lcc}
\hline & BPH $(n=14)$ & PC $(n=12)$ \\
\hline $\begin{array}{l}\text { AGE } \\
\text { Mean } \pm \text { SD } \\
(\text { Range })\end{array}$ & $\begin{array}{l}67 \pm 7 \\
(56-79)\end{array}$ & $\begin{array}{c}70 \pm 7 \\
(68-84)\end{array}$ \\
\hline PSA & $3.02 \pm 1.58 \mathrm{ng} / \mathrm{mL}$ & $9.13 \pm 5.47 \mathrm{ng} / \mathrm{mL}$ \\
\hline Gleason score & n.a. & $\begin{array}{c}\text { Gleason }<8(60 \%) \\
\text { Gleason } \geq 8(40 \%)\end{array}$ \\
\hline Therapies & $\begin{array}{c}\text { 2/14 on } \alpha \text {-lytic agents } \\
\text { (no pts. on hormonal } \\
\text { treatment) }\end{array}$ & $\begin{array}{c}\text { No pts. on hormonal } \\
\text { treatment }\end{array}$ \\
\hline $\begin{array}{l}\text { Additional } \\
\text { notes }\end{array}$ & $\begin{array}{c}\text { 3/14 with chronic } \\
\text { inflammation }\end{array}$ & \\
\hline
\end{tabular}

reviewed and approved by the local board and funded by study Grants MIUR 2008NY72SJ and RBFR10URHP.

2.2. Enzymatic Assays. Unprocessed samples were split into three parts, one for enzymatic activity, one for mRNA gene expression studies, and one sent to the pathologist for confirmation. One hundred milligrams of intact tissue was exposed to physiological concentrations of different $\mathrm{H}_{3}$ labeled compounds (Sigma-Aldrich), to explore the capability of these cells to metabolize these substances, in serumfree buffering medium under controlled temperature and atmosphere. One hundred microliters of media was collected at different time points ( $30 \mathrm{~min}, 1 \mathrm{~h}, 2 \mathrm{~h}, 4 \mathrm{~h}, 8 \mathrm{~h}$, and $16 \mathrm{~h}$ ) after incubation of various $\mathrm{H}_{3}$-labeled steroids and total lipids were extracted with $400 \mu \mathrm{L}$ Folch reagent (chloroform/methanol: $2: 1 \mathrm{vol} / \mathrm{vol}$ ), vortexed, and spun at $14,000 \times \mathrm{g}$ for $5 \mathrm{~min}$. The organic phase was collected and evaporated in a speedvac. Dried extracts were redissolved in $40 \mu \mathrm{L}$ of ethanol and spotted onto TLC plates (Whatman). The plates were developed twice in chloroform-ethyl acetate $(4: 1 \mathrm{vol} / \mathrm{vol})$. Steroid metabolites were quantified using a BioScan AR-2000 Imaging System (Bioscan). Bidimensional acquisition of $\beta$ emission was obtained using dedicated software (Bioscan).

2.3. Reverse Transcriptase-Polymerase Chain Reaction (RT$P C R)$. RNA was extracted from tissue samples using GenElute TM Mammalian Total RNA Miniprep kit according to the manufacturer's instructions (Sigma-Aldrich). $1 \mu \mathrm{g}$ of total RNA was retrotranscribed in a total volume of $50 \mu \mathrm{L}$ using random primers (F. Hoffmann-La Roche, Basel, Switzerland) and M-MLV reverse transcriptase (Invitrogen, Carlsbad, CA) and used as template for real-time polymerase chain reaction. Real-time quantitative PCRs (qPCR) was performed using $2 \mu \mathrm{L}$ of $\mathrm{cDNA}, 18 \mu \mathrm{M}$ for each primer, $5 \mu \mathrm{M}$ for probe (Applied Biosystems), TaqMan GenEX Master Mix (Applied Biosystems), and iQCycler (Bio-Rad Laboratories) according to the manufacturer's instructions. $17 \beta$-HSD1, $17 \beta$-HSD2, $17 \beta$-HSD3, $17 \beta$-HSD4, $17 \beta$-HSD5, $17 \beta$-HSD7, $17 \beta$-HSD8, $17 \beta$-HSD10, aromatase, and beta-actin (actb) human primers were used. Data were analyzed using the standard curve method. The relative quantities of transcripts were calculated 

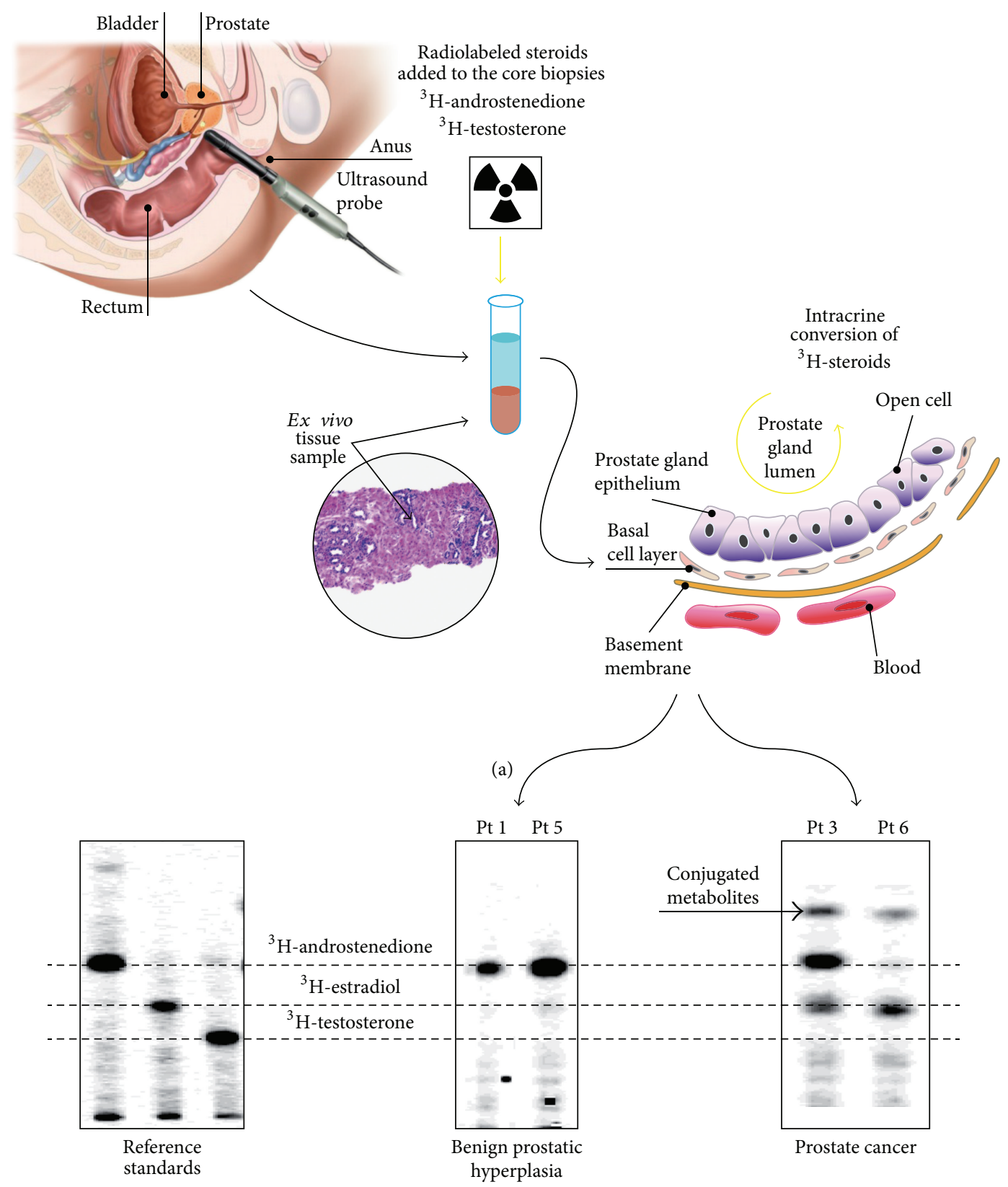

(b)

FIGURE 1: (a) Unique features of our "ex vivo" model: samples were histologically confirmed on-site, part of each sample was immediately dry frozen for subsequent RNA analysis, and part was fixed and embedded for paraffin section analysis. The core biopsies guaranteed tissue integrity that is crucial to maintain enzyme kinetics and directionality closer to what occurs in in vivo; specifically the stromal-to-epithelial interaction remains unaltered, allowing appraisal of the contribution of tumor microenvironment. (b) Examples of different TCL patterns (steroid fingerprint) in patients with BPH or PC (monodimensional development). Of notice is that patients with different stage PC exhibit different steroid maps (patient 3 had a Gleason 6, while patient 6 a Gleason 8). Incubation time: 24 hours. BPH: benign prostatic hyperplasia; PC: prostate cancer.

from triplicate samples after normalization of the data against the housekeeping gene (actb).

2.4. Data Analysis and Statistical Methods. Differences between experimental groups were analyzed by the Student's $t$-test and chi-square test. Spearman's correlation coefficients were used to assess the relationship between experimental variables. Multiple comparisons were performed using a oneway ANOVA and Turkey's post hoc test. The test was twosided and $P \leq 0.05$ was considered significant. All analyses were performed using SPSS version 17.0 PC version (SPSS Inc., Chicago, IL, USA). 


\section{Results}

Clinical features of the enrolled patients are reported in Table 1. None of the recruited patients were taking steroid hormones or chemotherapy or received previous external beam radiation. Two $\mathrm{PBH}$ patients were on $\alpha$-adrenolytic treatment of lower urinary tract symptoms.

To determine the efficiency whereby various precursors undergo intraprostatic conversion to more potent steroids, an operation protocol has been designed as follows: normal and cancerous cells derived from bioptic specimens were exposed to physiological concentrations of different labelled compounds (herein described for ${ }^{3} \mathrm{H}$-androstenedione or ${ }^{3} \mathrm{H}$ testosterone) as described in methods. Enzymatic products were then separated by two-dimensional TLC (2D-TLC) by sequential use of two customized mobile phases in order to discriminate molecular compounds that may differ for a single atom of hydrogen. The $2 \mathrm{D}$ procedure started with a classical monodimensional TLC (as shown in Figure 1) that, when necessary, could be followed by a further separation based on the affinity with the second mobile phase run orthogonally to the first one. Because it is unlikely that two molecules will be similar in two distinct repartition properties, molecules are more effectively separated in $2 \mathrm{D}$ TLC than in 1D-TLC. As a result the products of hormone metabolism spread out across the whole chromatographic surface. In respect to the current analysis of androstenedione/testosterone conversion into estrogens or testosterone a 1D-TLC was sufficient to discriminate the various steroids. Subsequent scanning of the developed TLC sheet, by means of the Argon-Methane enhanced $\beta$-emission scanner, will allow the identification of substrate-product(s) emitting spots and the measurement of CPM from each spot. Bioscan software renders an image that is equivalent to a "metabolic fingerprint" of each prostatic specimen (Figure 1(b)).

A representative pattern of conversion that has been observed in BPH and in PC is shown in Figure 1(b). When PC samples were exposed to ${ }^{3} \mathrm{H}$-androstenedione the most frequent observed pattern was estradiol conversion (66\%), while $17 \%$ of tissue revealed testosterone formation and the remaining $17 \%$ showed no conversion activity (Figure 2 ). On the contrary the majority of BPH samples showed no conversion $(60 \%)$ or estrogen formation (30\%); only $10 \%$ of samples exhibited some testosterone production. When PC samples were exposed to ${ }^{3} \mathrm{H}$-testosterone, the majority (66\%) revealed estrogen formation or no conversion (34\%); none showed formation of conjugated byproducts. On the contrary in $\mathrm{BPH}$ samples $60 \%$ of subjects showed formation of conjugated steroids, while estrogen formation was observed in $22 \%$ and $18 \%$ exhibited no conversion (Figure 2).

The synthesis of testosterone from precursor molecules occurs via a well-established sequence of reversible reactions. Since androgen levels may be affected by both changes in synthetic and degradative enzyme expression, gene expression analyses of $17 \beta$-HSD types 5 and 7 isoenzymes with predominant reductase activity versus types 4,8 , and 10 with a predominant oxidase activity were performed in $\mathrm{BPH}$ and PC (Figure 3). Compared to normal tissue, $\mathrm{BPH}$ showed a significant lower expression level of the $17 \beta$-HSD types 5, 7, and 10 enzymes, consistently with a reduced de novo enzymatic production of androgens; in respect to PC, all samples showed lower levels of expression for all enzymes, compared to BPH tissue. This finding is in apparent contrast with the enzymatic conversion activities reported in Figure 2. Indeed, PC showed a predominant reduction in the expression of the $17 \beta$-HSDs with reductase activity compared to oxidizing ones, when compared with $\mathrm{BPH}$ or normal tissue.

Moreover none of the PC samples expressed $17 \beta$-HSD type 2, compared to $\mathrm{BPH}$, while maintaining an efficient expression of $17 \beta$-HSD type 3 enzymes and aromatase transcripts (Figure 4).

In Figure 5 estradiol and testosterone generation in all samples (compared to the maximum conversion achieved set to 10 ) is reported. Despite the relatively small cohort number, a greater enzymatic activity is seen in PC samples with a high grade Gleason score compared to low Gleason score (histology) $(P<0.05)$.

\section{Discussion}

Prostate cancer is considered a "hormone-dependent" disease because the prostate requires testicular androgens for its secretory function and cancerous cells retain this sensitivity to androgens. Cell growth and survival of early stage prostate cancer can in fact respond to androgens and this evidence is the background of the androgen-deprivation therapy (ADT). Although plasma concentrations of testosterone have been shown to decrease by more than $90 \%$ following castration, androgen levels in prostate cancer tissues decreased only by $50-60 \%$, suggesting the importance of in situ androgen production in prostate cancers $[6,15,16]$. In addition, $\mathrm{ADT}$ is associated with severe systemic adverse events such as cardiac, metabolic, hepatic, bone, sexual, and cognitive complications that eventually lead to an increased mortality rate compared to the risk related to prostatic cancer itself $[3,4]$. ADT is successful until the tumour enters an androgenrefractory state, leading to the failure of such long-term strategy.

So far it has been impossible to associate high levels of circulating androgens with the progression of prostate cancer [7]. This is in strong agreement with the decline of plasmatic testosterone with age, which would ultimately lead to an inverse relationship between circulating androgens and the risk of developing prostate cancer. As a matter of fact, intratissual levels of sex steroids may diverge from their plasmatic counterpart due to enzymatic equipment that can interconvert and metabolize steroids [9, 10, 15]. The local steroid metabolism is therefore the main determinant of the intraprostatic hormonal profile. Androgens level variation as sole determinants of prostate cancer development and progression has led to neglecting the evidence that the onset of malignancy is accompanied by an estrogen-sensitive condition where tumour growth and spread is stimulated and maintained by an increase in the cellular levels of androgen aromatization into estrogens, in a manner similar to that described in breast cancer $[6,11]$. Moreover, animal models 

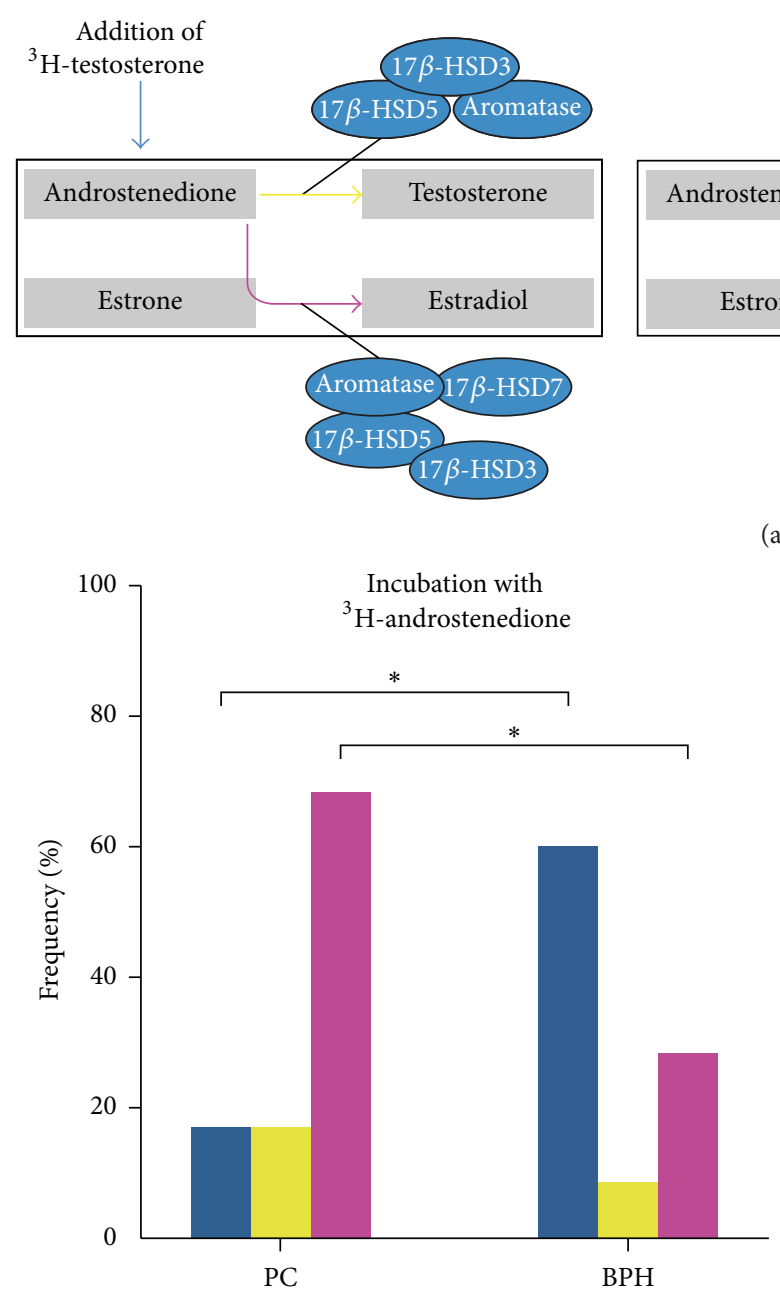

No conversion

Conversion to testosterone

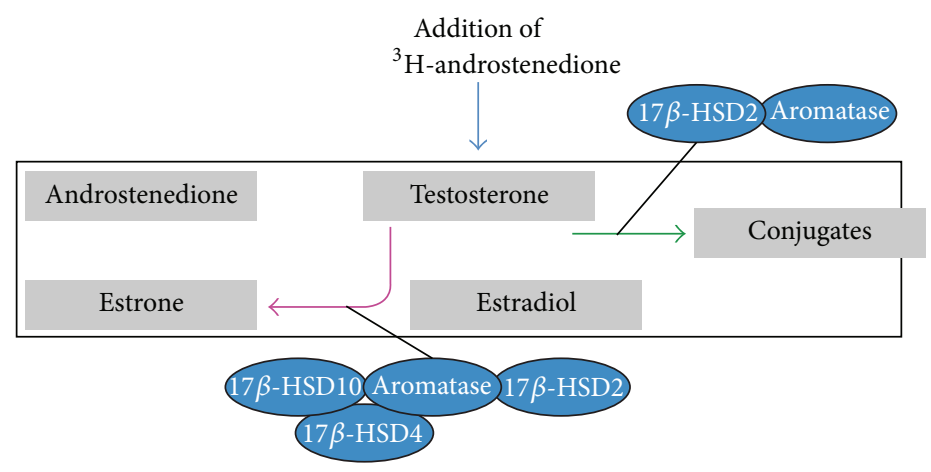

(a)

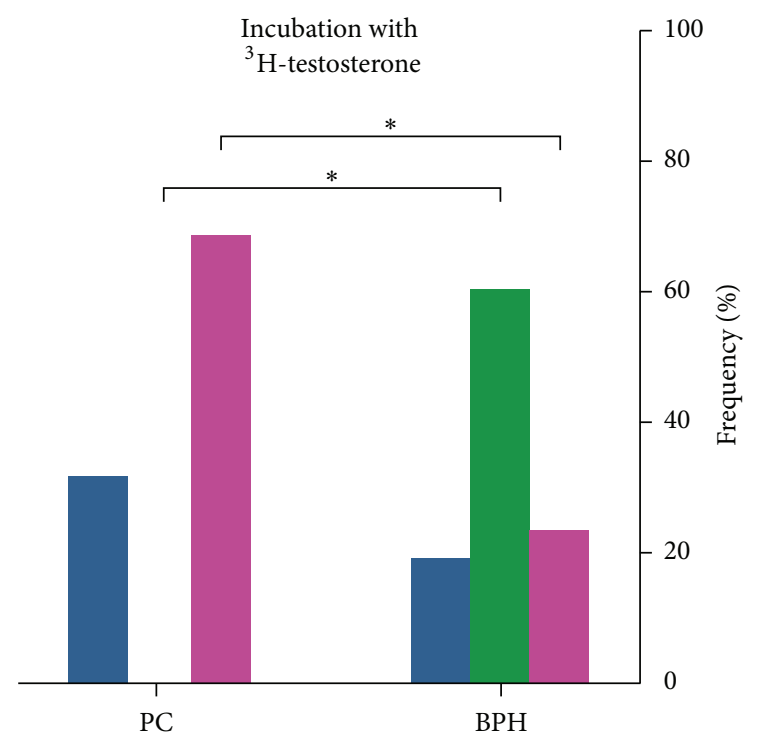

Conversion to estrone + estradiol

Formation of conjugated metabolites

(b)

Figure 2: (a) Schematic representation of the different pathways evaluated by TLC byproducts formation after exposure to ${ }^{3} \mathrm{H}$ androstenedione or ${ }^{3} \mathrm{H}$-testosterone. (b) Frequency of the major pattern of conversions observed during incubation in all subjects (more than one conversion reaction can be observed in the same subject). ${ }^{*} P<0.05$; BPH: benign prostatic hyperplasia $(n=14)$; PC: prostate cancer $(n=12)$.

have clearly showed that supraphysiological levels of estrogens and androgens are each separately capable of altering the normal growth of the prostate, but individually they do not induce prostatic malignancy. As neither hormone by itself is able to induce malignant changes in the prostate, the balance between sex steroids is critical in inducing premalignant and malignant lesions. In this respect, an altered profile of prostatic enzymes that metabolize steroids has to be invoked in the acquisition of aggressiveness of prostate cancer. At present, we barely know the exact molecular mechanisms underlining the progression of the prostatic disease and the acquisition of the metastatic behaviour.

Classical monitoring factors of prostate cancer, such as PSA levels, stadiation, and Gleason score, are losing their reliability in the attempt to discriminate among multiple stages of the disease. New prognostic and diagnostic markers are needed. Our goal was to develop novel methods to acquire data on the enzymatic profile (and its changes) in intact prostate tissue in order to characterize the history of the disease with respect to follow-up, medical, surgical, and radiotherapeutic procedures.

In addition, there is a recent growing interest toward several metabolites that could be bioactivated into more active steroids with high affinity binding to androgen receptor (AR) or estrogen receptor (ER) [17]. An interesting observation is that androgenic activity of the Cl1-keto forms of $\mathrm{A} 4, \mathrm{~T}$, and DHT are more androgenic than their respective 11-hydroxy forms [17]. This implicates that the activity of 


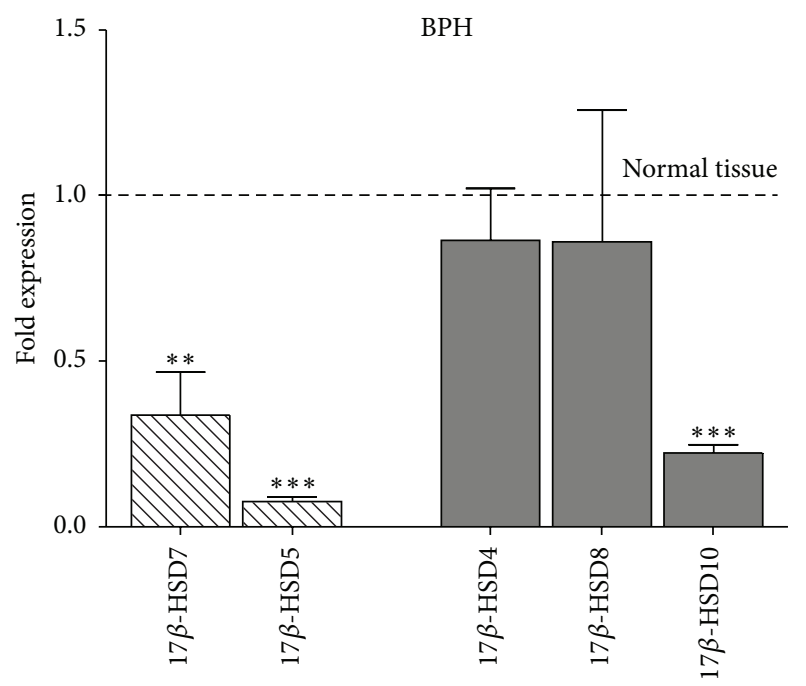

(a)

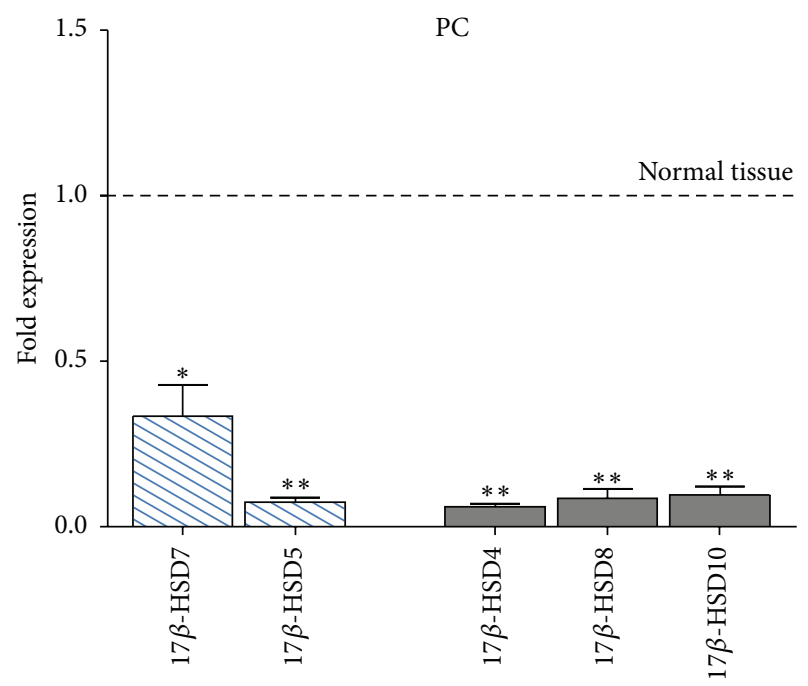

(b)

Figure 3: Quantitative gene expression in examined tissue relative to whole (normal) prostate tissue (fold change) of $17 \beta$-HSDs with predominant reductase activity (types 5 and 7 , yellow) versus $17 \beta$-HSDs with a predominant oxidase activity (types 4 , 8 , and 10, green). BPH: benign prostatic hyperplasia $(n=11)$; PC: prostate cancer $(n=7)$. Statistical significance is represented versus whole normal tissue ${ }^{* * *} P \leq 0.001 ;{ }^{* *} P<0.01$.

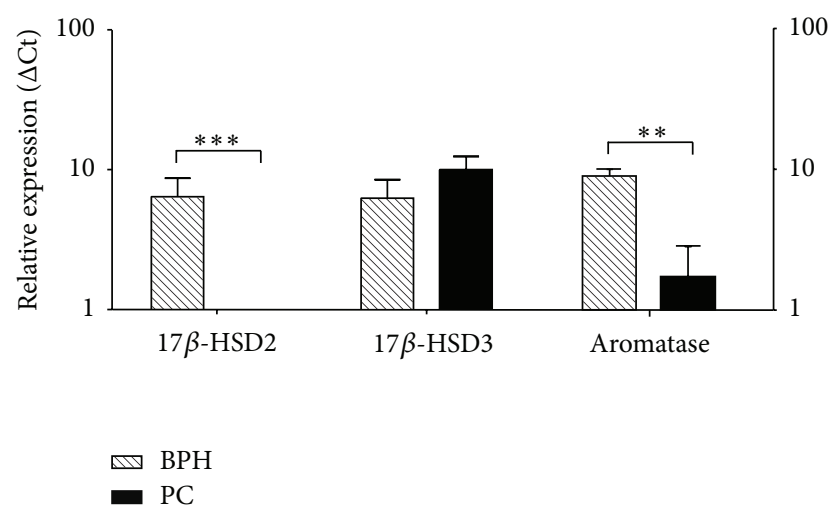

FiguRE 4: Quantitative gene expression in examined tissue relative to whole (normal) prostate tissue (normal set to 1 ) of $17 \beta$-HSD type 2 and type 3 and aromatase. BPH: benign prostatic hyperplasia $(n=$ $11)$; PC: prostate cancer $(n=7)$.

the 11 $\beta$-HSDs family, which interconvert 11-hydroxy and 11-keto steroids, could be another regulatory point in the activation or inactivation of 11-hydroxy and 11-keto androgens in the prostate [17]. The involvement of 11b-HSD in prostate physiology deserves future studies as it has already been shown to be crucial in several clinical conditions $[18,19]$.

The most extensively studied intracrinology pathways, nowadays, are those related to the $17 \beta$-HSDs.

Precise measurements of relative enzymatic activity of this family are complicated by the requirement for optimal $\mathrm{pH}$ and nicotinamide adenine dinucleotide cofactors that requires intact cell models. In this respect we believe that our model has the advantage of assessing this in the best way. In androgen and estrogen metabolism, 17HSDs catalyze

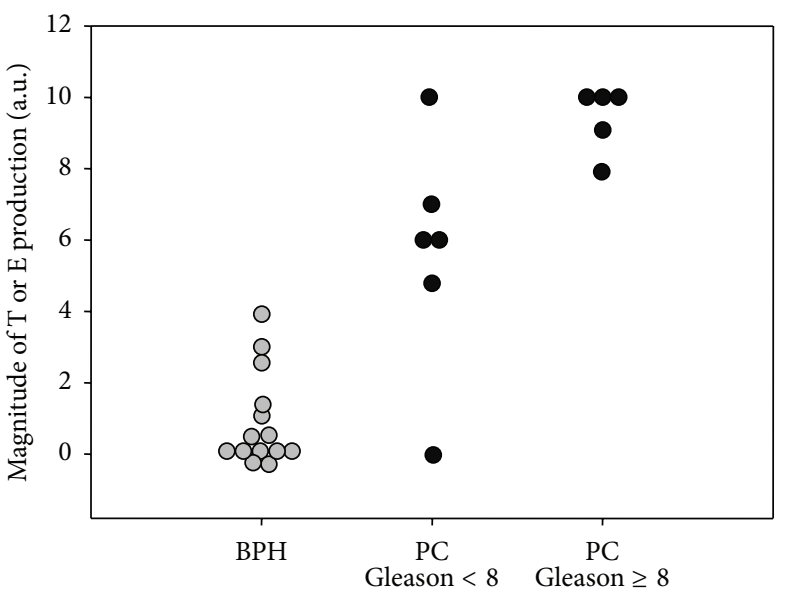

FIGURE 5: Correlation between conversion efficiency toward testosterone or estradiol and histological grading of tissue samples; $\mathrm{BPH}$ : benign prostatic hyperplasia (gray circle, $n=14$ ); PC: prostate cancer (solid circle, $n=12$ ).

the reactions between the active $17 \beta$-hydroxysteroids and less active 17-ketosteroids. At present, several 17HSD isoenzymes have been characterized [20]; specifically types $1,3,5$, and 7 are reductive enzymes, whereas types $2,4,8,10$, and 11 are oxidative enzymes. Very recently Fankhauser and colleagues showed that upregulation of 17HSD17B is the predominant source of signalling androgens in hormone refractory prostate cancer, much greater than either the socalled "backdoor" or the "5- $\alpha$ dione" pathway [14].

Our findings document that in an ex vivo model of intact prostate tissue, an enhanced reductive pathway is a feature of $\mathrm{PC}$, compared to BPH. These data are consistent with those 
by Nakamura et al., demonstrating that in human prostate cancer $17 \beta$-HSD 5 immunoreactivity was detectable in $77 \%$ of cases with a stronger staining correlated to more advanced clinical stages (TNM stage pT3 versus pT2) [21].

The current idea is that the progression of prostate cancer in the setting of castrate androgen levels is not due to the development of an androgen insensitive tumour clone but rather to the fact that the cancer has evolved mechanisms to escape systemic androgen deprivation while still taking advantage of signalling through AR $[6,13-15,22]$.

The recognition of the fact that intratumoural androgen synthesis and activity are biologically relevant and that overexpression of the AR is a consistent feature of prostate cancer progression has led to the development of several new therapeutic approaches. One example is the utilization of abiraterone acetate, an inhibitor of $17 \alpha$-hydroxylase and C17, 20 lyase (CYP17A1) for advanced CRPC treatment. Blockade of CYP17A1 activity by abiraterone suppresses androstenedione, dehydroepiandrosterone (DHEA), testosterone, and oestradiol formation, as well as other metabolites [23]. We believe that our approach could be useful to monitor intracrinology changes of patients under novel enzyme-targeting drugs.

In the innovation of the methodological approach, we rely on several facts: (1) because of the high sensitivity of the detection system, the enzymatic activity can be determined using exceedingly low amounts of labelled compounds mimicking the in vivo conditions; (2) the number of cells required to test the activity is also very small allowing test on biopsies; (3) enzymatic activity is assessed in the optimal $\mathrm{pH}$ and nicotinamide adenine dinucleotide cofactors concentration; (4) tissues can be subsequently processed for other uses.

The present work, however, has also some limitations. One limitation is that we did not measure DHT levels that could have been altered by both type 1 and type 2 5- $\alpha$ reductase activity [21]. However, taking into account that patients with $\mathrm{BPH}$ or prostate cancer often use specific $5-\alpha$ inhibitors (2 out of 14 in our series) this was not possible. Another limitation of the present study is that we did not address the role $17 \beta$-HSD6. This enzyme has been recently considered the backdoor pathway of DHT synthesis in patients undergoing ADT [24] since it exerts a key role in androstanediol bioactivation to the downstream pathway leading to DHT formation from adrenal steroids [25]. However, in vitro assessment of 17 $\beta$-HSD6 activity is very problematic and most of studies simply rely only on mRNA expression, that, as we have shown, does not necessarily reflect protein levels and activities. In addition, a recent study revealed that $17 \beta$-HSD6 is expressed in ER $\beta$ positive epithelial cells of the human prostate but that in prostate cancers of Gleason grade higher than 3, both ER $\beta$ and $17 \beta$ HSD6 become undetectable [26], suggesting that formation of $3 \beta$-Adiol via $17 \beta$ HSD6 from DHT could be considered an important growth regulatory pathway, but not a marker of prostate cancer aggressiveness [26]. Finally, future studies are ongoing to characterize the phase two metabolism of steroids derivatives that are understudied and could be a future target for castrate-resistant prostate cancer [27].

In summary, we developed an easy tool to profile individual intraprostatic enzymatic activity (steroid map or fingerprint) by characterizing conversion pathways in an intact tissue environment from fresh biopsies. Using this novel approach we found that $17 \beta$-HSD-isoenzymes and aromatase activity in prostate tissue cultures correlate with biological behaviour. This approach could be a novel useful tool for clinical monitoring of novel enzyme-targeting drugs.

\section{Conflict of Interests}

The authors declare that there is no conflict of interests regarding the publication of this paper.

\section{Authors' Contribution}

Daniele Gianfrilli and Silvia Pierotti equally contributed to the paper.

\section{Acknowledgment}

The study was supported by Ministry of Research MIUR Grants PRIN 2008 no. 2008NY72SJ and FIRB 2010 no. RBFR10URHP.

\section{References}

[1] J. M. Correas, E. Drakonakis, A. M. Isidori et al., "Update on ultrasound elastography: miscellanea. Prostate, testicle, musculo-skeletal," European Journal of Radiology, vol. 82, no. 11, pp. 1904-1912, 2013.

[2] A. Aversa, A. M. Isidori, D. Gianfrilli et al., "Are subjects with erectile dysfunction aware of their condition? Results from a retrospective study based on an Italian free-call information service," Journal of Endocrinological Investigation, vol. 27, no. 6, pp. 548-556, 2004.

[3] A. M. Isidori, J. Buvat, and G. Corona, "A critical analysis of the role of testosterone in erectile function: from pathophysiology to treatment-a systematic review," European Urology, vol. 65, no. 1, pp. 99-112, 2014.

[4] F. Saad, A. Aversa, A. M. Isidori, L. Zafalon, M. Zitzmann, and L. Gooren, "Onset of effects of testosterone treatment and time span until maximum effects are achieved," European Journal of Endocrinology, vol. 165, no. 5, pp. 675-685, 2011.

[5] R. J. Santen, H. Brodie, E. R. Simpson, P. K. Siiteri, and A. Brodie, "History of aromatase: saga of an important biological mediator and therapeutic target," Endocrine Reviews, vol. 30, no. 4, pp. 343-375, 2009.

[6] G. P. Risbridger, I. D. Davis, S. N. Birrell, and W. D. Tilley, "Breast and prostate cancer: more similar than different," Nature Reviews Cancer, vol. 10, no. 3, pp. 205-212, 2010.

[7] K. K. Tsilidis, R. C. Travis, P. N. Appleby et al., "Interactions between genome-wide significant genetic variants and circulating concentrations of insulin-like growth factor 1 , sex hormones, and binding proteins in relation to prostate cancer risk in the National Cancer Institute breast and prostate cancer cohort consortium," The American Journal of Epidemiology, vol. 175, no. 9, pp. 926-935, 2012.

[8] P. Soronen, M. Laiti, S. Törn et al., "Sex steroid hormone metabolism and prostate cancer," Journal of Steroid Biochemistry and Molecular Biology, vol. 92, no. 4, pp. 281-286, 2004. 
[9] F. Labrie, A. Dupont, J. Simard, V. Luu-The, and A. Belanger, "Intracrinology: the basis for the rational design of endocrine therapy at all stages of prostate cancer," European Urology, vol. 24, supplement 2, pp. 94-105, 1993.

[10] F. Labrie, V. Luu-The, S. Lin et al., "The key role of $17 \beta$ hydroxysteroid dehydrogenases in sex steroid biology," Steroids, vol. 62, no. 1, pp. 148-158, 1997.

[11] G. P. Risbridger, J. J. Bianco, J. S. Ellem, and J. S. McPherson, "Oestrogens and prostate cancer," Endocrine-Related Cancer, vol. 10, no. 2, pp. 187-191, 2003.

[12] W. A. Ricke, S. J. McPherson, J. J. Bianco, G. R. Cunha, Y. Wang, and G. P. Risbridger, "Prostatic hormonal carcinogenesis is mediated by in situ estrogen production and estrogen receptor alpha signaling," The FASEB Journal, vol. 22, no. 5, pp. 1512-1520, 2008.

[13] P. Härkönen, S. Törn, R. Kurkela et al., "Sex hormone metabolism in prostate cancer cells during transition to an androgen-independent state," Journal of Clinical Endocrinology and Metabolism, vol. 88, no. 2, pp. 705-712, 2003.

[14] M. Fankhauser, Y. Tan, G. Macintyre et al., "Canonical androstenedione reduction is the predominant source of signalling androgens in hormone refractory prostate cancer," Clinical Cancer Research, 2014.

[15] J. A. Locke, E. S. Guns, A. A. Lubik et al., "Androgen Levels increase by intratumoral de novo steroidogenesis during progression of castration-resistant prostate cancer," Cancer Research, vol. 68, no. 15, pp. 6407-6415, 2008.

[16] A. Mizokami, E. Koh, H. Fujita et al., "The adrenal androgen androstenediol is present in prostate cancer tissue after androgen deprivation therapy and activates mutated androgen receptor," Cancer Research, vol. 64, no. 2, pp. 765-771, 2004.

[17] K. Storbeck, L. M. Bloem, D. Africander, L. Schloms, P. Swart, and A. C. Swart, "11 $\beta$-Hydroxydihydrotestosterone and 11ketodihydrotestosterone, novel C19 steroids with androgenic activity: A putative role in castration resistant prostate cancer?" Molecular and Cellular Endocrinology, vol. 377, no. 1-2, pp. 135146, 2013.

[18] A. M. Isidori, G. A. Kaltsas, L. Perry, J. M. Burrin, G. M. Besser, and J. P. Monson, "The effect of growth hormone replacement therapy on adrenal androgen secretion in adult onset hypopituitarism," Clinical Endocrinology, vol. 58, no. 5, pp. 601-611, 2003.

[19] S. Pierotti, L. Gandini, A. Lenzi, and A. M. Isidori, "Prereceptorial regulation of steroid hormones in bone cells: insights on glucocorticoid-induced osteoporosis," Journal of Steroid Biochemistry and Molecular Biology, vol. 108, no. 3-5, pp. 292-299, 2008.

[20] J. Adamski and F. J. Jakob, "A guide to $17 \beta$-hydroxysteroid dehydrogenases," Molecular and Cellular Endocrinology, vol. 171, no. 1-2, pp. 1-4, 2001.

[21] Y. Nakamura, T. Suzuki, M. Nakabayashi et al., "In situ androgen producing enzymes in human prostate cancer," EndocrineRelated Cancer, vol. 12, no. 1, pp. 101-107, 2005.

[22] H. I. Scher, G. Buchanan, W. Gerald, L. M. Butler, and W. D. Tilley, "Targeting the androgen receptor: improving outcomes for castration-resistant prostate cancer," Endocrine-Related Cancer, vol. 11, no. 3, pp. 459-476, 2004.

[23] G. Attard, A. H. Reid, T. A. Yap et al., "Phase I clinical trial of a selective inhibitor of CYP17, abiraterone acetate, confirms that castration-resistant prostate cancer commonly remains hormone driven," Journal of Clinical Oncology, vol. 26, no. 28, pp. 4563-4571, 2008.
[24] F. Ishizaki, T. Nishiyama, T. Kawasaki et al., "Androgen deprivation promotes intratumoral synthesis of dihydrotestosterone from androgen metabolites in prostate cancer," Scientific Reports, vol. 3, Article ID 1528, 2013.

[25] J. L. Mohler, M. A. Titus, S. Bai et al., "Activation of the androgen receptor by intratumoral bioconversion of androstanediol to dihydrotestosterone in prostate cancer," Cancer Research, vol. 71, no. 4, pp. 1486-1496, 2011.

[26] S. Muthusamy, S. Andersson, H. Kim et al., "Estrogen receptor $\beta$ and $17 \beta$-hydroxysteroid dehydrogenase type 6 , a growth regulatory pathway that is lost in prostate cancer," Proceedings of the National Academy of Sciences of the United States of America, vol. 108, no. 50, pp. 20090-20094, 2011.

[27] K. M. McNamara, Y. Nakamura, Y. Miki, and H. Sasano, "Phase two steroid metabolism and its roles in breast and prostate cancer patients," Frontiers in Endocrinology, vol. 4, article 116, 2013. 


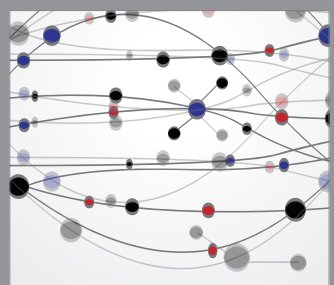

The Scientific World Journal
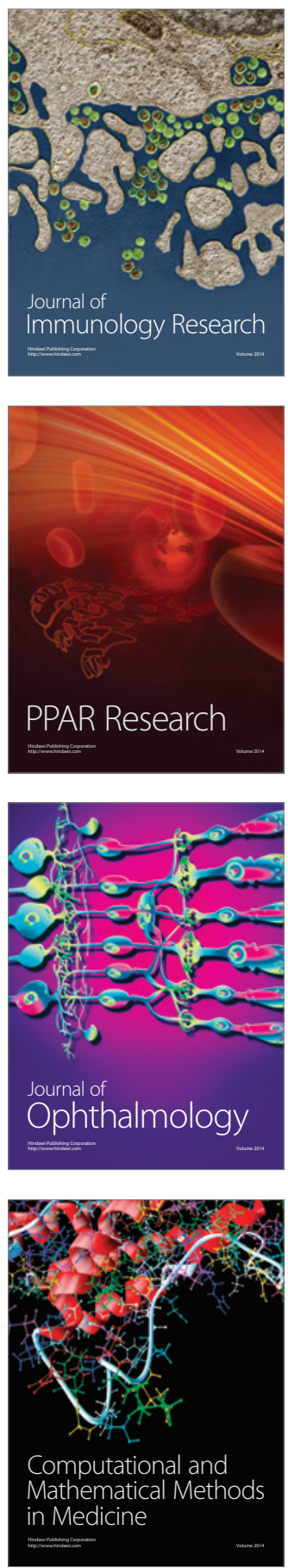

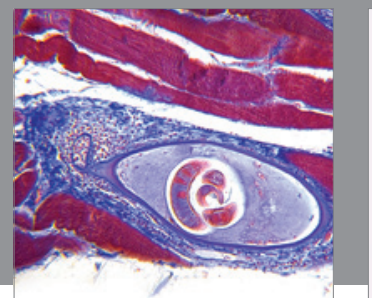

Gastroenterology

Research and Practice
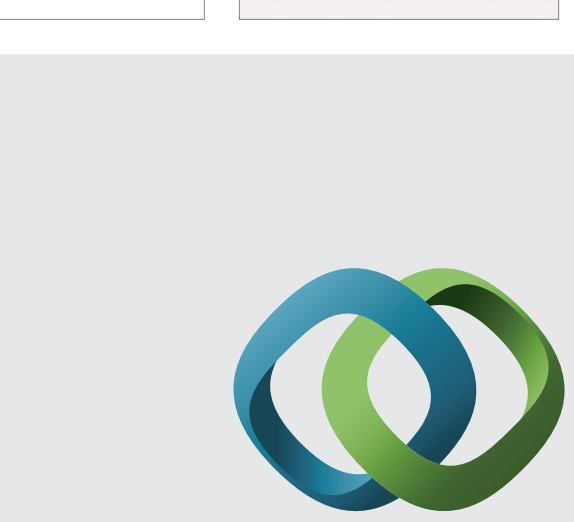

\section{Hindawi}

Submit your manuscripts at

http://www.hindawi.com
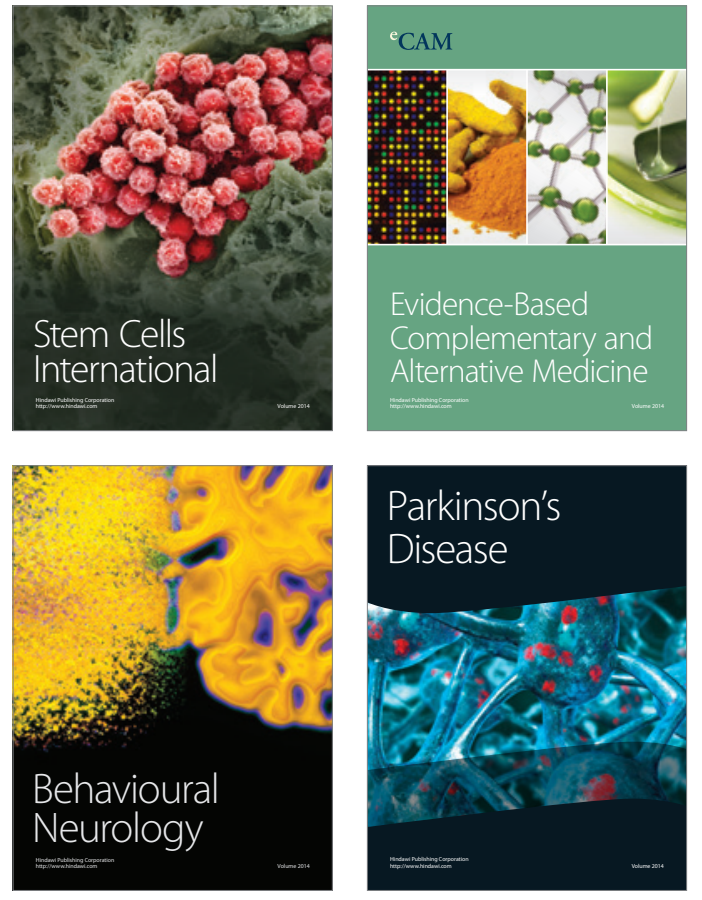
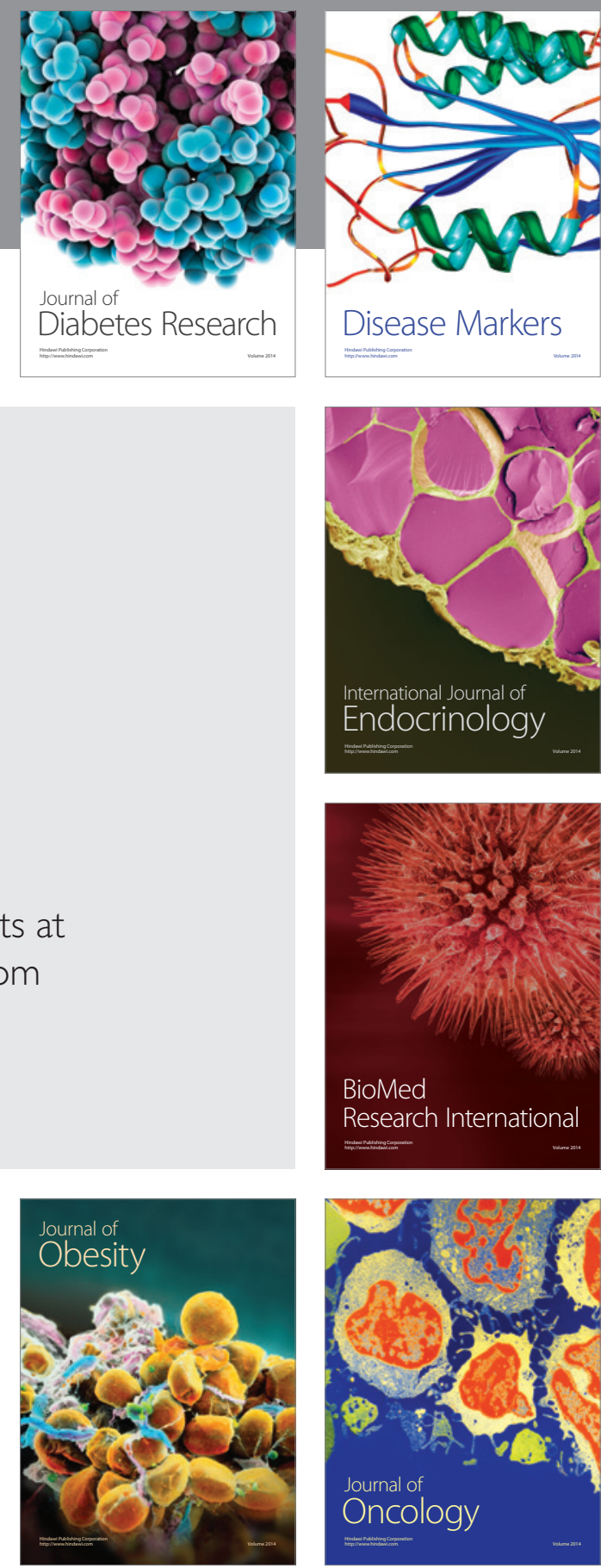

Disease Markers
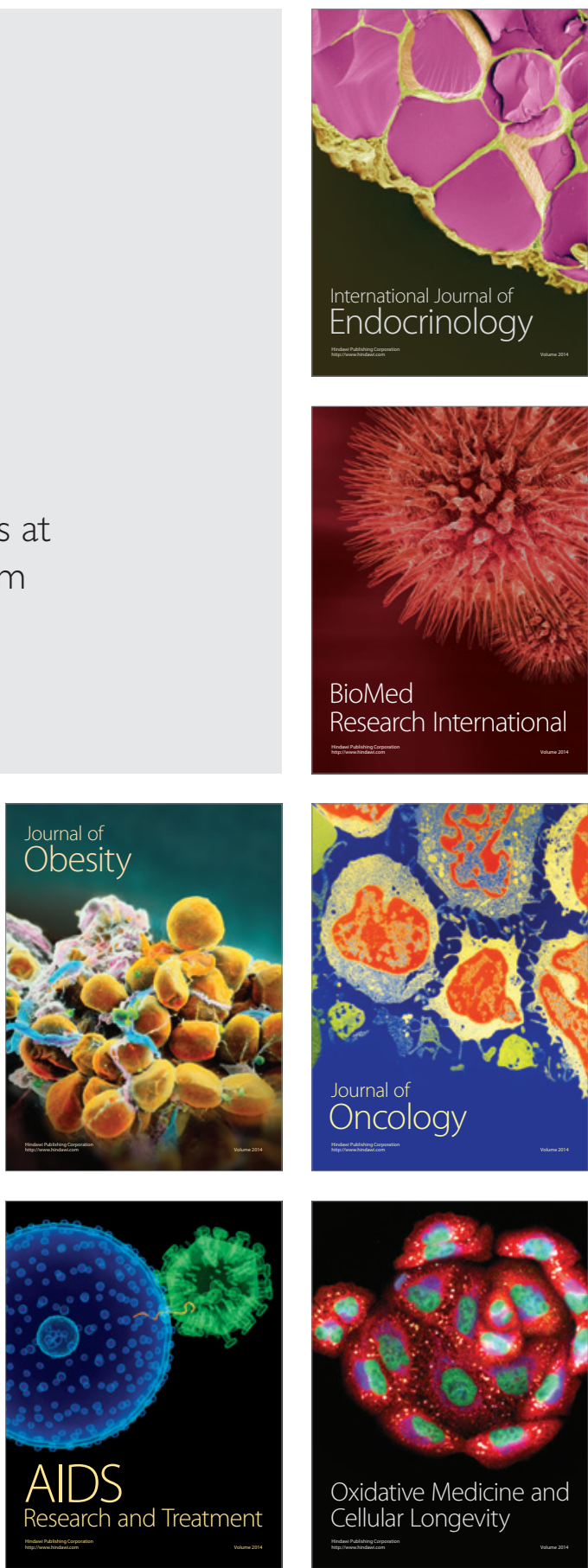